\title{
Coexpression of myofibroblast and macrophage markers: novel evidence for an in vivo plasticity of chorioamniotic mesodermal cells of the human placenta
}

\author{
Sung-Su Kim ${ }^{1}$, Roberto Romero, ${ }^{1,2}$, Jung-Sun Kim ${ }^{1,3,4}$, Asad Abbas ${ }^{1}$, Jimmy Espinoza ${ }^{1,4}$, Juan Pedro Kusanovic ${ }^{1}$, \\ Sonia Hassan ${ }^{1,4}$, Bo Hyun Yoon ${ }^{5}$ and Chong Jai Kim ${ }^{1,3}$
}

Human chorioamniotic membranes generate temporary but large mucosal surfaces. Due to lack of fetal vessels, macrophages represent the only subset of immunocytes of fetal origin available in the chorioamniotic mesodermal layer. This layer contains two distinct groups of cells: the fibroblasts/myofibroblasts and the macrophages; however, the relative contribution of these two cell populations has been a point of contention. In addressing various discrepancies, we hypothesized that cells in the chorioamniotic mesodermal layer have plasticity. Immunophenotyping of these cells using a panel of antibodies (CD14, CD68, CD163, HLA-DR, type I procollagen, $\alpha$-smooth muscle actin, desmin, vimentin) revealed coexpression of both myofibroblast and macrophage markers. The proportion of CD14+ macrophages was higher in inflamed chorioamniotic membranes $(P<0.05)$. Cells immunoreactive to the macrophage markers showed nuclear expression of PU.1, a hematopoietic cell-specific transcription factor. Furthermore, treatment with proinflammatory cytokines (IL-1 $\beta$ and TNF $\alpha$ ) or Toll-like receptor-4 overexpression upregulated PU.1 mRNA expression in chorioamniotic mesodermal cells. Overexpression of PU.1 in chorionic mesodermal cells increased the expression of CD14 mRNA and protein. A reporter gene assay and chromatin immunoprecipitation demonstrated binding of PU.1 to the CD14 promoter region. This study reports that chorioamniotic mesodermal cells display plasticity ranging from overt transformation of fibroblast/myofibroblast to macrophages, and that PU.1 plays a role in macrophage differentiation. Chorioamniotic mesodermal cells are another novel example of phenotypic switching between fibroblast/myofibroblast and macrophage. The findings reported herein suggest that the plasticity of mesodermal cells is an effective mechanism of the chorioamniotic membranes to manage several biological needs, such as mucosal immune defense and the maintenance/disruption of physical integrity, with a limited pool of cells.

Laboratory Investigation (2008) 88, 365-374; doi:10.1038/labinvest.3700749; published online 28 January 2008

KEYWORDS: chorioamniotic membranes; mesodermal layer; myofibroblast; macrophage; PU.1

In most cases, human mucosal surfaces are the initial foci of infections in the body. ${ }^{1}$ The resulting mucosal inflammation is associated with the infiltration of leukocytes; the type of infiltrating leukocytes depends on the phase of inflammation as well as the type of invading pathogens. ${ }^{2,3}$ Leukocytes generally infiltrate subepithelial spaces, such as the dermis of the skin or lamina propria of the gastrointestinal tract, by mobilization from the vascular bed. ${ }^{3-5}$ However, in the case of human chorioamniotic membranes, a highly specialized interface between the mother and fetus, such mobilization becomes problematic. Composed of amnion, chorion, and thin layers of deciduas, the membranes generate temporary but large stretches of two mucosal surfaces: the amnion facing the amniotic cavity and the chorion facing the uterine cavity. ${ }^{6}$ A feature unique to the free human chorioamniotic membranes is the absence of fetal vessels during all but a limited period of development, ${ }^{7}$ thus making mobilization of leukocytes from fetal circulation virtually impossible.

Intra-amniotic infection induces an acute and robust inflammatory reaction of the chorioamniotic membranes. This reaction, termed acute chorioamnionitis, is a primary cause of preterm deliveries and associated perinatal morbidities. ${ }^{8-10}$

\footnotetext{
${ }^{1}$ Perinatology Research Branch, NICHD/NIH/DHHS, Bethesda, MD and Detroit, MI, USA; ${ }^{2}$ Center for Molecular Medicine and Genetics, Wayne State University School of Medicine, Detroit, MI, USA; ${ }^{3}$ Department of Pathology, Wayne State University School of Medicine, Detroit, MI, USA; ${ }^{4}$ Department of Obstetrics and Gynecology, Wayne State University School of Medicine, Detroit, MI, USA and ${ }^{5}$ Department of Obstetrics and Gynecology, Seoul National University College of Medicine, Seoul, Korea Correspondence: Dr R Romero, MD or Dr CJ Kim, MD, PhD, Perinatology Research Branch, NICHD/NIH/DHHS, Wayne State University/Hutzel Women's Hospital, 3990 John R, 4th Floor, Detroit, Ml 48201, USA. E-mail: prbchiefstaff@med.wayne.edu, cjkim@med.wayne.edu
}

Received 28 September 2007; revised 12 December 2007; accepted 12 December 2007 
In acute chorioamnionitis, the maternal neutrophils extravasated from decidual vessels are exclusive infiltrates into the membranes. ${ }^{11}$ On the fetal side, macrophages resident in the chorioamniotic mesodermal layer represent the only population of immune cells available. The chorioamniotic mesodermal layer is positioned between amnion epithelial cells and chorionic trophoblasts. Several observations indicate that the cells of this layer, populated in abundant collagenous stroma, participate in various biological processes, including the production of collagen, inflammatory mediators, and uterotonic prostaglandins. This strongly suggests that their functions are closely linked to vital aspects of human pregnancy and parturition. ${ }^{12,13}$

The chorioamniotic mesodermal layer is known to contain two distinct groups of cells: the fibroblasts/myofibroblasts and the macrophages. ${ }^{14}$ However, the proportion of fibroblasts/myofibroblasts and macrophages within this layer has been a point of controversy and intrigue. ${ }^{7,14-18}$ Although discrepancies among research findings may be due, in part, to differences in patient populations, the sampling sites in the membranes, and the markers or techniques employed during analyses, inconsistencies among previous studies led us to hypothesize that plasticity of the cells could be an alternative explanation. Our assumption was particularly based on the changes and regional variations in the morphology of the chorioamniotic mesodermal cells encountered in the zone of altered morphology (ZAM) ${ }^{19}$ the chorioamniotic membranes exposed to meconium, and those affected with chorioamnionitis-all of which contain many cells with the macrophage phenotype. Myofibroblasts have a hybrid phenotype between fibroblasts and smooth muscle cells. They are also capable of synthesizing both collagen and matrix metalloproteinases (MMPs), and thus important in tissue remodeling. ${ }^{14,20}$ Phenotypic switching between fibroblast and myofibroblast is common. ${ }^{21}$ Macrophages, on the other hand, are important effector immunocytes orchestrating innate and adaptive immune responses. ${ }^{22}$

As an adjustable biological container of the fetus, the chorioamniotic membranes should possess enough physical strength and flexibility necessary for functions like immune defense and rupture to ensure that delivery takes place. ${ }^{12,13}$ In this respect, transdifferentiation of fibroblasts/myofibroblasts into macrophages, or vice versa, within a pool of chorioamniotic mesodermal cells would be an effective mechanism. In this study, therefore, we investigated evidence for the plasticity of chorioamniotic mesodermal cells.

\section{MATERIALS AND METHODS} Tissue Samples

Snap-frozen or formalin-fixed, paraffin-embedded chorioamniotic membrane rolls were retrieved from the bank of biological specimens at the Perinatology Research Branch, National Institute of Child Health and Human Development (NICHD). The samples were collected from the following study groups: (1) the preterm chorioamniotic membranes with and without acute chorioamnionitis $(n=10$ and $n=10$, respectively), (2) the chorioamniotic membranes obtained from women in labor at term $(n=10)$, and (3) meconiumstained membranes at term $(n=5)$. The use of tissue for research purposes was approved by the Institutional Review Boards of both Wayne State University and the NICHD.

\section{Immunohistochemistry/Immunofluorescence}

Snap-frozen or paraffin-embedded sections were obtained from each specimen and placed on a silanized slide. Single or double immunohistochemical staining with a panel of antibodies to CD14 (Vector Laboratories, Burlingame, CA, USA), CD68 (PG-M1; Dako, Carpinteria, CA, USA), CD163 (Vector Laboratories), type I procollagen (SP1.D8; Developmental Studies Hybridoma Bank, University of Iowa, Iowa City, IA, USA), $\alpha$-smooth muscle actin ( $\alpha$ SMA; Vector Laboratories), desmin (Dako), vimentin (LabVision Corporation, Fremont, CA, USA), Ki-67 (Dako), and PU.1 (BD Biosciences, Mississauga, ON, Canada) was performed using automatic immunostainers (Ventana Benchmark: Ventana Medical Systems Inc., Tucson, AZ, USA; Dako Universal autostainer). DAB (3-3'diaminobenzidine) and Vector blue served as chromogens. CD14 expression for each test group was quantified by digital image analysis using Image-Pro Plus software (Media Cybernetics Inc., Bethesda, MD, USA). Images from 10 random high-power fields $(\times 400)$ on a bright field microscope were captured for each slide. Immunofluorescence staining was performed using antibodies to CD14, CD68, CD163, HLA-DR (Dako), and $\alpha$ SMA.

\section{Chromogenic In Situ Hybridization}

Chromogenic in situ hybridization (CISH) was performed on $5 \mu \mathrm{m}$-thick paraffin-embedded sections of the fetal membranes. The reaction was conducted using a Y chromosomespecific probe (DYZ1; Zymed Laboratories Inc., San Francisco, CA, USA), according to the manufacturer's recommendations. Briefly, deparaffinized sections were incubated for $15 \mathrm{~min}$ at $100^{\circ} \mathrm{C}$ in Spot-Light Tissue Heat Pretreatment Buffer (Zymed Laboratories Inc.) and washed immediately in distilled water two times. Sections underwent enzymatic digestion at $37^{\circ} \mathrm{C}$ for $10 \mathrm{~min}$ with the Spot-Light Tissue Pretreatment Enzyme (Zymed Laboratories Inc.), followed by washing and dehydration in a series of graded alcohols. After allowing to air dry, $15 \mu \mathrm{l}$ of the probe was added to the slides. Denaturation was performed at $95^{\circ} \mathrm{C}$ for $5 \mathrm{~min}$, followed by hybridization for $10 \mathrm{~h}$ at $37^{\circ} \mathrm{C}$ in a humidified chamber. Y chromosome immunodetection was observed with the CISH centromeric detection kit (Zymed Laboratories Inc.). Counterstaining was done with hematoxylin.

\section{Chorionic Mesodermal Cell Culture}

Primary chorionic mesodermal cells were isolated from term chorioamniotic membranes. After removal of the amnion, the surface of the chorionic mesodermal layer was treated 
with $0.25 \%$ collagenase for $40 \mathrm{~min}$ at $37^{\circ} \mathrm{C}$ in a shaking incubator. Alternatively, whole chorion-decidua was sequentially treated with dispase II $(2.4 \mathrm{U} / \mathrm{ml}$; Roche Diagnostic, Basel, Switzerland) and collagenase for an additional $1.5 \mathrm{~h}$ with intervening meticulous scraping to remove decidua and chorionic trophoblasts. The chorioamniotic mesodermal cells in the collagenase solution were then harvested and propagated in McCoy's 5A or $\alpha$ MEM containing $10 \%$ FBS and antibiotics. All transfection experiments were completed using $\alpha$ MEM. Possible contamination of trophoblasts was evaluated by flow cytometry for cytokeratin-7. For all of the subsequent experiments, the cells between passages 5 and 9 were used. The cells were treated with IL-1 $\beta$ and TNF $\alpha$, or transfected with full-length TLR-4 for $24 \mathrm{~h}$. RT-PCR analysis for PU.1 mRNA expression was done using the primers for PU.1 (sense: 5'-CAGCTCAGATGAGGAGGAG-3', antisense: $5^{\prime}$-CTTGGACGAGAACTGGAAG- $3^{\prime}$ ) and $\beta$-actin (sense: $5^{\prime}$ GCCAACACAGTGCTGTCTGG-3', antisense: $5^{\prime}$-TACTCCT GCTTGCTGATCCA- ${ }^{\prime}$ ).

\section{Cloning of PU.1 and Transfection}

Total RNA was isolated from human placenta. RT-PCR was performed using a One-step RT-PCR kit (QIAGEN, Valencia, CA, USA). The primers used were $5^{\prime}$-GAATTCAGGCGTG CAAAATGGAAGGG-3' (sense), and 5'-CTCGAGTCAGT GGGGCGGGTGGCGCC-3' (antisense). The PCR reaction was composed of initial denaturation at $94^{\circ} \mathrm{C}$ for $5 \mathrm{~min}, 35$ cycles of $94^{\circ} \mathrm{C}$ for $1 \mathrm{~min}, 50^{\circ} \mathrm{C}$ for $1 \mathrm{~min}, 72^{\circ} \mathrm{C}$ for $1 \mathrm{~min}$, and $72^{\circ} \mathrm{C}$ for $10 \mathrm{~min}$. The PCR product $(750 \mathrm{bp}$ ) was cloned into a pCRII-TOPO vector (Invitrogen, Carlsbad, CA, USA). The sequence-confirmed insert was subsequently cloned in pcDNA3.1 (Invitrogen) for transient expression studies.

\section{Real-Time Quantitative Reverse Transcription-PCR}

Real-time quantitative reverse transcription-PCR (qRT-PCR) was performed with TaqMan Gene Expression Assays (Hs00169122_g1, Applied Biosystems, Foster City, CA, USA) to assess the mRNA expression of CD14 mRNA. Reverse transcription of the DNase-treated total RNA was performed using a SuperScript III reverse transcriptase (Invitrogen) and oligo(dT) primers. The reaction was analyzed with the 7500 Fast Real-Time PCR System (Applied Biosystems). $\beta$-Actin (Applied Biosystems) was used as an internal control.

\section{Immunoblotting}

The PU.1 transfected cells were lysed using RIPA buffer. A $25 \mu \mathrm{g}$ portion of protein was electrophoresed in a $12 \%$ SDSpolyacrylamide gel and subsequently transferred onto the nitrocellulose membrane. The primary antibody used was anti-CD14 (Santa Cruz Inc., Santa Cruz, CA, USA). Signals were detected with enhanced chemiluminescence.

\section{Flow Cytometry}

Primary chorionic mesodermal cells harvested from the fetal membranes were subjected to flow cytometric analysis. The cells were labeled with murine monoclonal anti-CD163 antibody conjugated with phycoerythrin (BD Biosciences, San Jose, CA, USA). For cytokeratin-7 or CD68 labeling, the cells were permeabilized using permeabilizing solution 2 (BD Biosciences), and incubated with murine monoclonal anticytokeratin-7 (Dako) or murine monoclonal anti-CD68, followed by reaction with FITC-conjugated horse anti-mouse immunoglobulin. Expression of CD163 and cytokeratin-7 was analyzed using FACSAria (BD, Franklin Lakes, NJ, USA).

\section{CD14 Promoter Region Analysis and Reporter Gene Assay}

The TESS: Transcriptional Element Search System (http:// www.cbil. upenn.edu/tess) was used to predict putative transcription factor binding sites. Luciferase fusion plasmids containing the DNA sequence from $-600 \mathrm{bp}$ upstream of the human CD14 gene (pGL-600 construct; from -600 to $+1 \mathrm{bp}$ relative to the translation start site being +1 ) to various lengths of $5^{\prime}$-short downstream regulatory sequences were constructed. The $5^{\prime}$-deletion construct, pGL-600, was generated by PCR with the upstream primer at -600 bp bearing an XhoI site and the downstream primers bearing a HindIII site. The PCR fragment was cloned into a pGL3-Basic (Promega, Madison, WI, USA). Primary chorioamniotic mesodermal cells were transiently transfected with each plasmid using Lipofectamine 2000 transfection reagent (Invitrogen). For the adjustment of transfection efficiency, pCH110 (Amersham Biosciences, Piscataway, NJ, USA) containing the $\beta$-galactosidase gene was used. Luminometry was performed $48 \mathrm{~h}$ after transfection.

\section{Chromatin Immunoprecipitation Assay}

Primary chorionic mesodermal cells transiently transfected with PU.1 were used for a chromatin immunoprecipitation (ChIP) assay. Twenty-four hours after transfection, chromatin was cross-linked by the addition of formaldehyde to the culture medium at a final concentration of $1 \%$ and incubated for $10 \mathrm{~min}$ at $37^{\circ} \mathrm{C}$. Cells were washed twice with PBS containing a protease inhibitor cocktail and resuspended in ChIP lysis buffer (50 mM HEPES pH 7.5, $140 \mathrm{mM} \mathrm{NaCl}, 1 \%$ Triton X-100, $0.1 \%$ sodium deoxycholate, protease inhibitors). The lysate was sonicated to yield fragments ranging from 200 to $1000 \mathrm{bp}$. The samples were precleared at $4^{\circ} \mathrm{C}$ with recombinant protein A/G-agarose beads (Santa Cruz Biotechnology Inc., Santa Cruz, CA, USA). A $100 \mu$ l portion of precleared lysate diluted in immunoprecipitation buffer (0.01\% SDS, $1.1 \%$ Triton X-100, $1.2 \mathrm{mM}$ EDTA, $16.7 \mathrm{mM}$ Tris-HCl, $\quad \mathrm{pH} \quad 8.1$, and $167 \mathrm{mM} \mathrm{NaCl})$ was immunoprecipitated with $10 \mu \mathrm{g}$ of anti-PU.1 antibody (Santa Cruz Biotechnology Inc.) overnight at $4^{\circ} \mathrm{C}$. The complexes were collected by incubating with recombinant protein A/G-agarose beads for $2 \mathrm{~h}$. After washing and elution, formaldehyde cross-linking was reversed with $6 \mathrm{~h}$ incubation at $65^{\circ} \mathrm{C}$. DNA was precipitated after a phenol/chloroform extraction and dissolved in $50 \mu \mathrm{l}$ of TE buffer. PCR reaction 
mixtures contained $2 \mu \mathrm{l}$ of the immunoprecipitated chromatin sample with the following primers spanning the CD14 promoter region in $20 \mu \mathrm{l}$ of total volume. The CD14 primers, $5^{\prime}$-CAGAATCCTTCTGTTACGG-3' (sense) and 5'-TGGTCG ATAAGTCTTCCGAA-3' (antisense), produce a $280 \mathrm{bp}$ product.

\section{Statistical Analysis}

Mann-Whitney $U$-test was used for the data analysis. SPSS version 12.0 (SPSS Inc., Chicago, IL, USA) was employed for statistical analysis.

\section{RESULTS}

\section{Immunophenotypes of Chorioamniotic Mesodermal Cells}

Chorioamniotic mesodermal cells are usually short spindle cells with relatively small, hyperchromatic nuclei and little cytoplasm. Immunoreactivity of these cells to various antigens, such as macrophage markers (CD14, CD163, CD68) and markers of smooth muscle differentiation (desmin and aSMA), was relatively weak compared to that of type I procollagen and vimentin (Figure 1a). In contrast, the chorioamniotic mesodermal cells close to the ZAM were characterized by vesicular nuclei, abundant cytoplasm, and strong immunoreactivity for CD14, CD163, procollagen, and desmin (Figure 1a). Cells double positive for CD163 and procollagen were readily found (Figure $1 \mathrm{~b}$ ). The macrophages of meconium-exposed chorioamniotic membranes were strongly immunoreactive for CD163, whereas nonreactive or weakly reactive to type I procollagen and desmin (Figure 1c). Using a Y chromosome-specific probe (DYZ1), CISH of fetal membranes containing ZAM from patients who delivered male babies $(n=3)$ demonstrated distinct intranuclear a
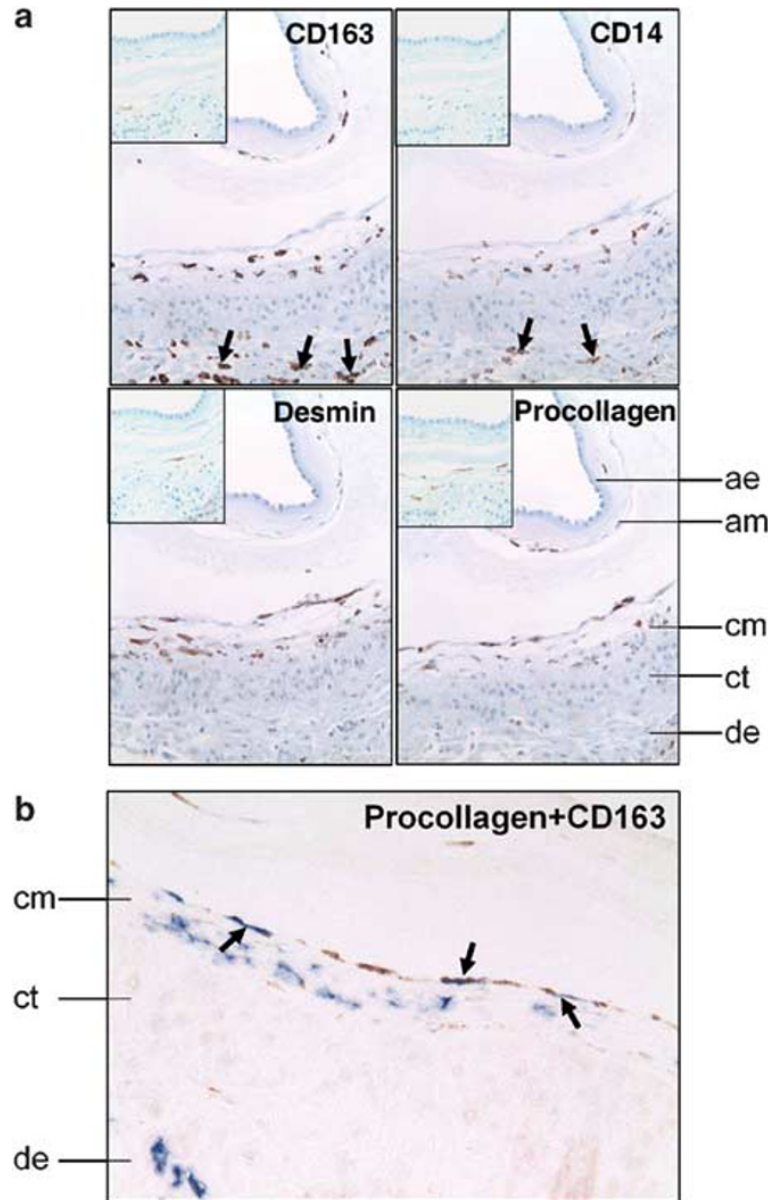

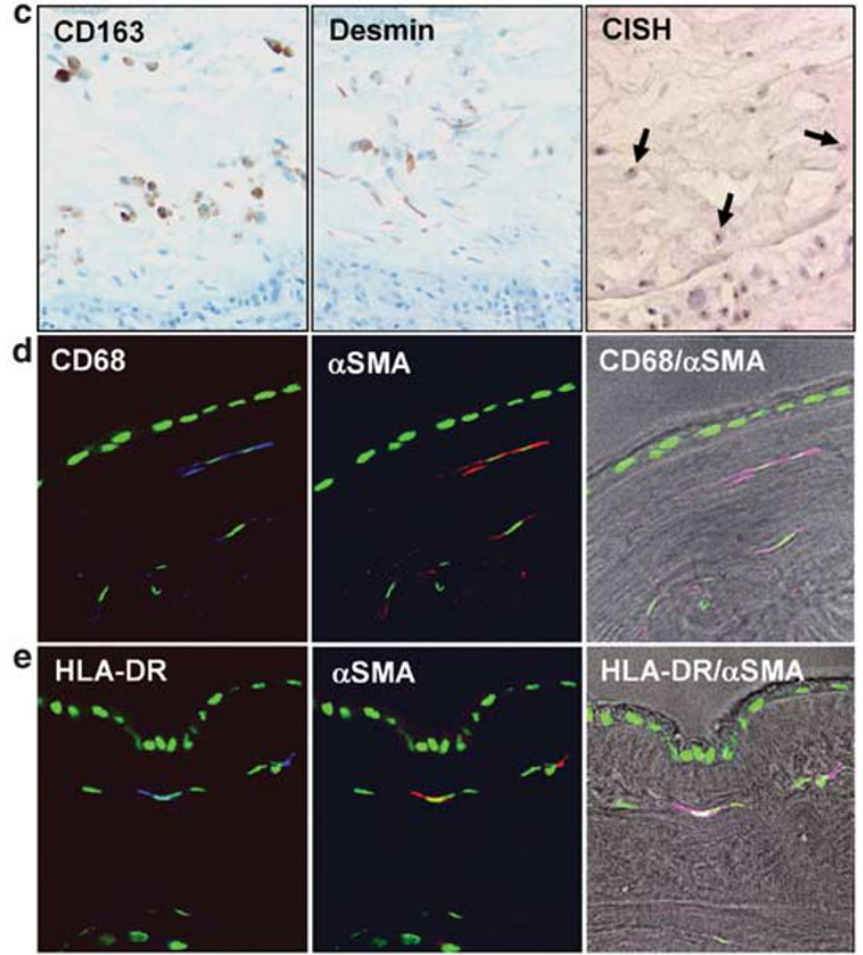

Figure 1 Immunophenotypes of representative chorioamniotic mesodermal cells. (a) The chorioamniotic mesodermal cells near the zone of altered morphology (ZAM) show frequent labeling of CD163, CD14, desmin, and procollagen. Decidual macrophages (arrows), negative to desmin, are in stark contrast. Insets show immunoreactivity for the markers in the same fetal membranes apart from the ZAM. ae, amnion epithelium; am, amnionic mesoderm; $\mathrm{cm}$, chorionic mesoderm; ct, chorionic trophoblasts; de, decidua. (b) Chorionic mesodermal cells showing coexpression of CD163 and type I procollagen on double immunohistochemical staining (brown: CD163-DAB, blue: type I procollagen-Vector blue). (c) Meconium-containing macrophages strongly positive for CD163. Those cells show weak and variable immunoreactivity to desmin. CISH using DYZ1 probe shows distinct nuclear hybridization signals in the chorioamniotic mesodermal cells of ZAM (arrows) indicating their fetal origin. (d) Immunofluorescence staining shows CD68 (blue; Alexa Fluor 633 ) and aSMA (red; Alexa Fluor 568) double-positive cells in the chorioamniotic mesoderm. Nuclei of the cells were stained green (Sytox green). (e) Scattered mesodermal cells are double positive for HLA-DR (blue; Alexa Fluor 633) and $\alpha$ SMA (red; Alexa Fluor 568). 
signals in the chorioamniotic mesodermal cells (Figure 1c), confirming their fetal origin. Immunofluorescence staining was done for further analysis of immunoreactivity of chorioamniotic mesodermal cells. In the chorioamniotic membranes of term labor cases, cells double positive for CD68 and $\alpha$ SMA or HLA-DR and $\alpha$ SMA were also noted (Figure 1d and e). Overall findings clearly show subsets of cells expressing both myofibroblast and macrophage markers.

Our assessment also considered the possibility of a change in the proportion of fibroblasts/myofibroblasts to macrophages with acute chorioamnionitis in the chorioamniotic mesodermal layer. The proportion of $\mathrm{CD} 14+$ macrophages in the chorioamniotic mesodermal layer was significantly higher in cases with acute chorioamnionitis than in those without acute chorioamnionitis $(P<0.05$; Figure $2 \mathrm{a}$ and $\mathrm{b})$. As the increase in macrophages could be the result of in situ proliferation or de novo transdifferentiation, Ki-67 immunostaining was also performed in selected cases; Ki-67 labeling of the mesodermal cells was rarely encountered (Figure 2c). To further assess the possibility of infiltration of maternal macrophages from decidua in acute chorioamnionitis, $\mathrm{CISH}$ using a $\mathrm{Y}$ chromosome-specific probe was performed. The cases from women who delivered male babies were used $(n=3)$. The reaction also confirmed that the chorioamniotic mesodermal cells, but not neutrophils, are of fetal origin (Figure 2d).

\section{Expression of PU.1 and its Binding to CD14 Promoter in Mesodermal Cells}

Increases in CD14 immunoreactivity of mesodermal cells in acute chorioamnionitis led us to study the expression of PU.1, a transcription factor specific to hematopoietic cells and essential for embryonic lymphoid and myeloid development. ${ }^{23,24}$ The goal was to determine if cells representing truly hematopoietic lineages were present. Many of the cells expressing CD163 showed positive nuclear labeling of PU.1 (Figure 3a).

For in vitro analysis, we performed a primary culture of chorioamniotic mesodermal cells. Initial primary chorionic mesodermal cells are composed of flat, substrate-adherent cells and cells with many cytoplasmic processes consistent with macrophages (Figure 3b). Initial primary cells contain a subset of CD163 + cells, but in subsequent passages become phenotypically homogeneous with the loss of CD163 + cells a
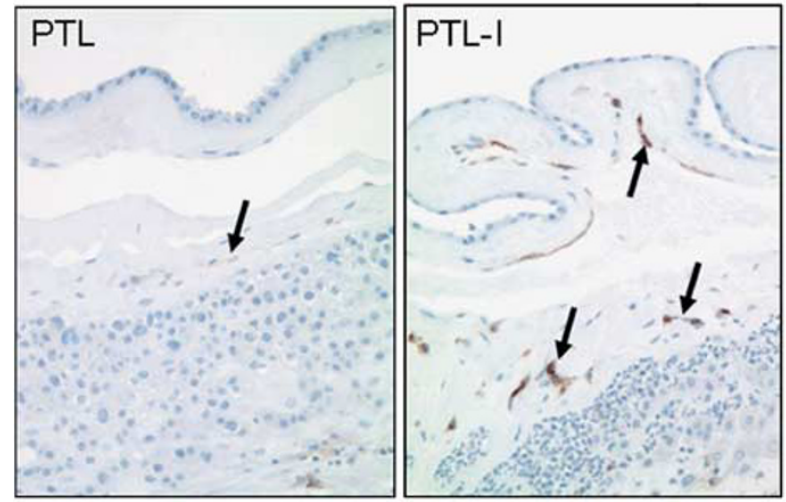

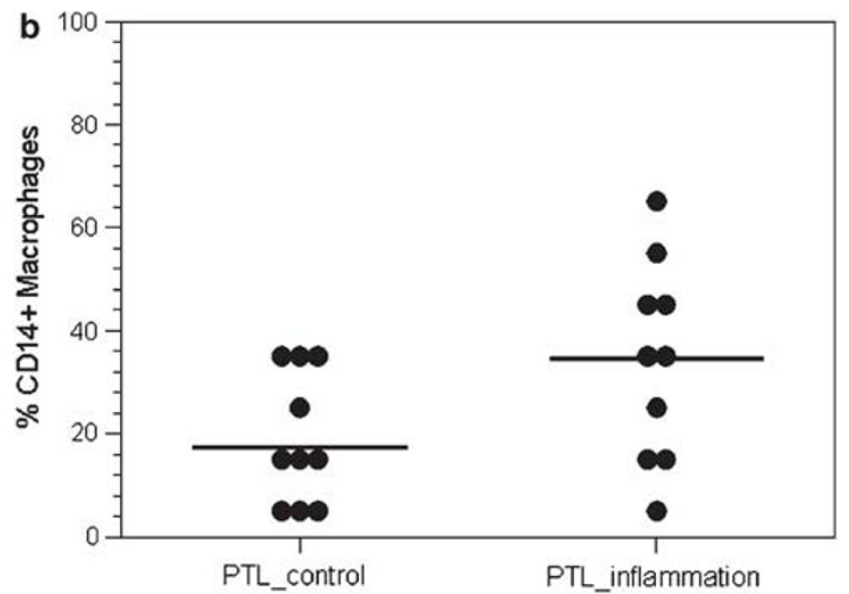

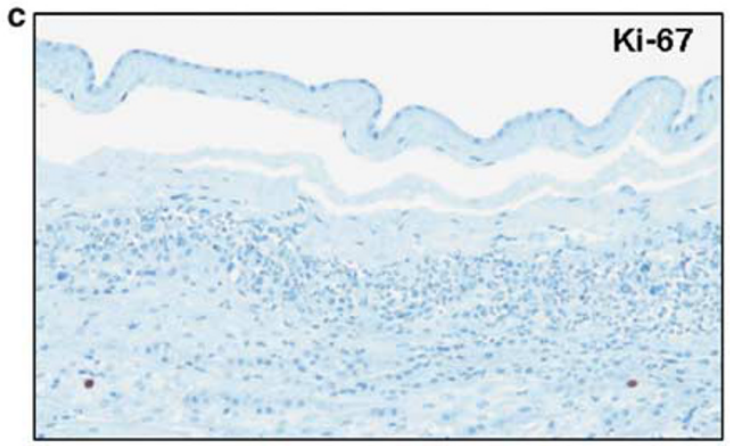

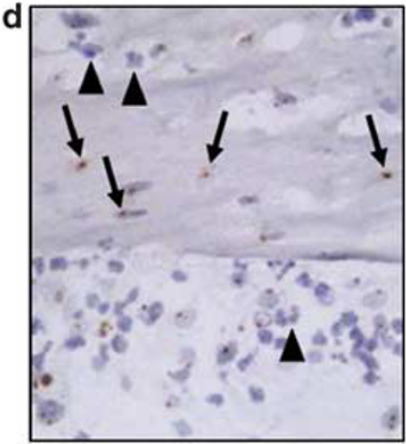

Figure 2 Phenotypic changes of the chorioamniotic mesodermal cells associated with acute chorioamnionitis. (a, b) The proportion of CD14 + macrophages is significantly higher in cases with acute chorioamnionitis $(P<0.05)$. PTL, preterm labor; PTL-I, preterm labor with acute chorioamnionitis. (c) Ki-67-positive mesodermal cells are not encountered in the fetal membranes with acute chorioamnionitis, whereas a couple of decidual cells show Ki-67 labeling. (d) CISH using a DYZ1 probe showing distinct intranuclear hybridization signals in the chorioamniotic mesodermal cells (arrows) but not in the infiltrating maternal neutrophils (arrowheads), in a case of acute chorioamnionitis. 

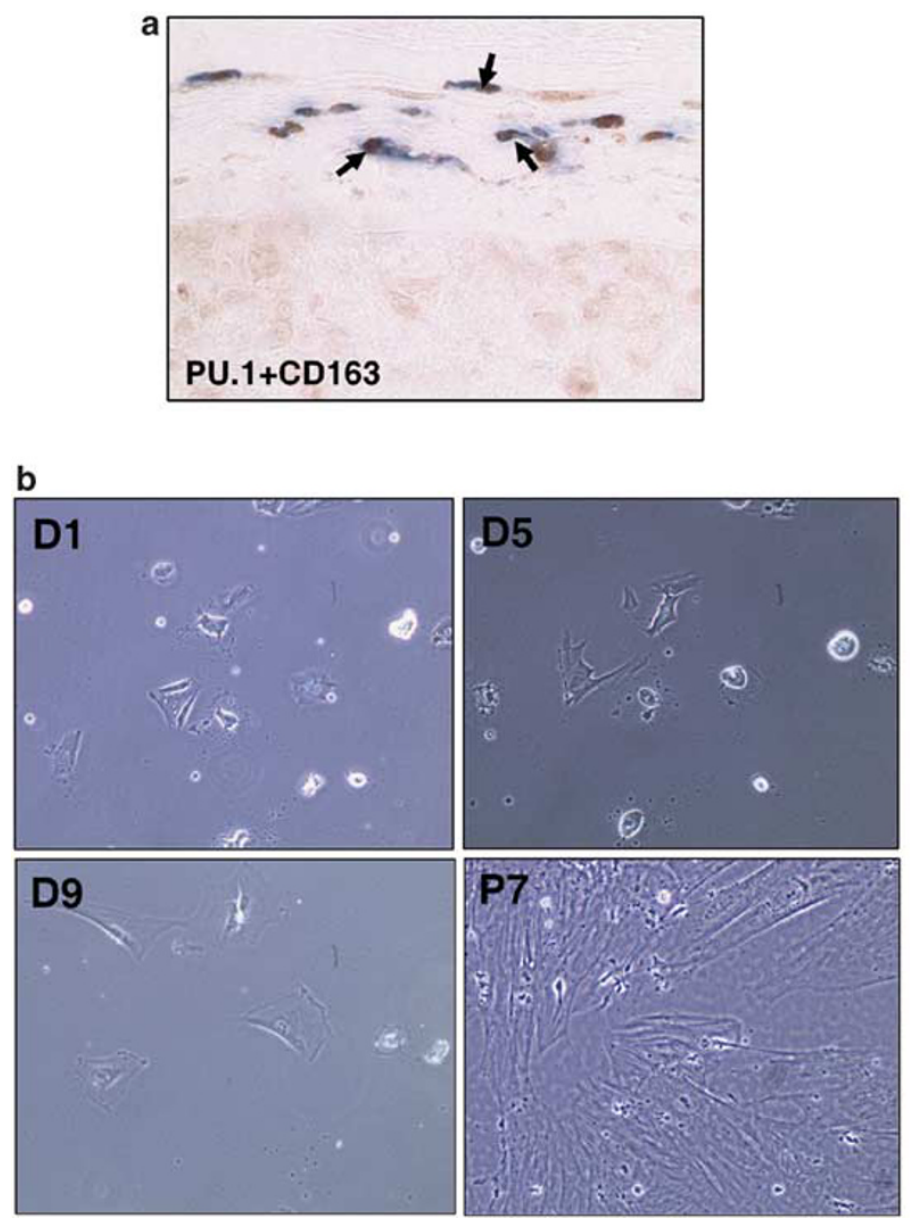
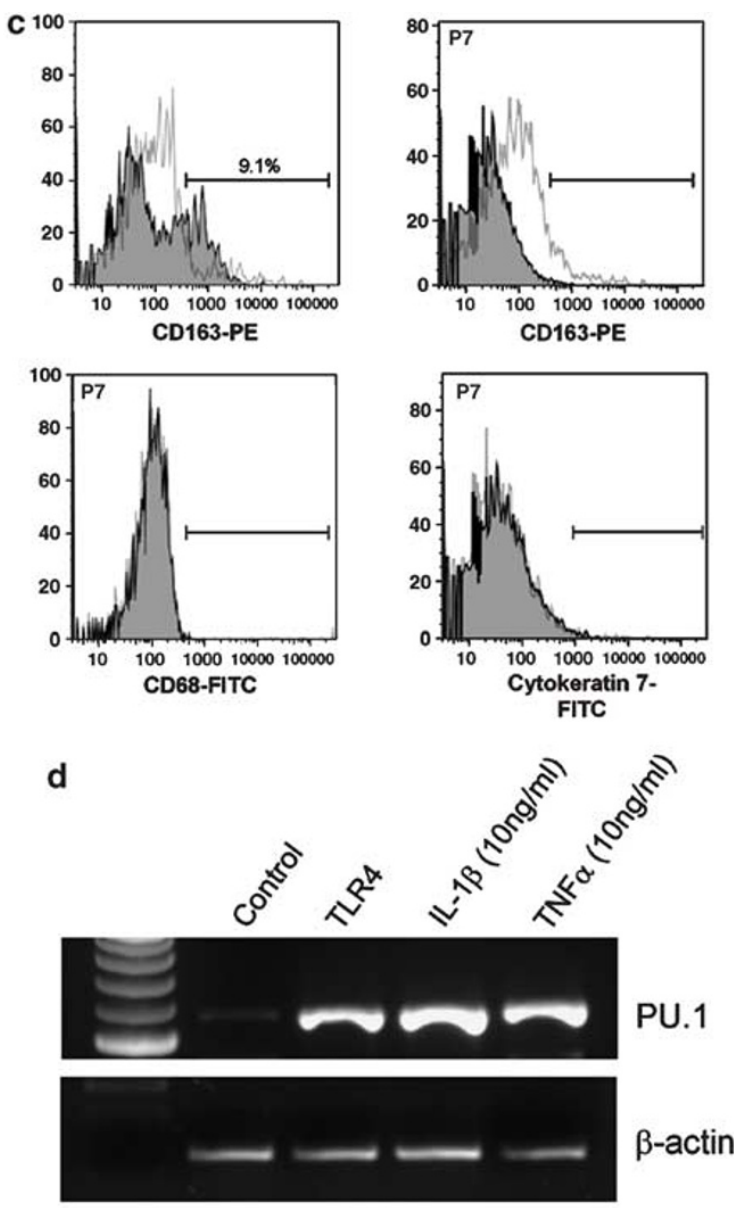

Figure 3 PU.1 expression and regulation in the chorioamniotic mesodermal cells. (a) Intranuclear PU.1 expression in the chorioamniotic mesodermal cells. Many of these cells are CD163 +. Double immunohistochemistry, PU.1 (brown: DAB), CD163 (blue: Vector blue). (b) Initial primary chorionic mesodermal cells are adherent and composed of mainly flat, polygonal cells and some cells with abundant cytoplasmic processes. The cells were obtained by $0.25 \%$ collagenase digestion of the surface of the chorioamniotic mesodermal layer. The cells become homogeneous with passages losing the cells with abundant cytoplasmic processes. D1: day 1, D5: day 5, D9: day 9, P7: passage 7. (c) Flow cytometric analysis of chorionic mesodermal cells. Initially harvested cells contain CD163 + cells, whereas the cells at passage 7 do not show significant expression of either CD163 or CD68. The cells are negative for cytokeratin-7, showing their nonepithelial phenotype. Open histograms represent isotype controls. (d) RT-PCR results showing a marked increase in PU.1 mRNA expression following treatment of primary chorionic mesodermal cells with IL-1 $\beta$ ( $10 \mathrm{ng} / \mathrm{ml})$ and TNF $\alpha$ (10 ng/ml) or transient transfection with full-length TLR-4 for $24 \mathrm{~h}$.

(Figure 3c). We then assessed whether a proinflammatory response is associated with changes in PU.1 expression by looking at the changes in PU.1 mRNA expression of the primary chorionic mesodermal cells. Treatment with IL-1 $\beta$ $(10 \mathrm{ng} / \mathrm{ml})$ and $\mathrm{TNF} \alpha(10 \mathrm{ng} / \mathrm{ml})$ or transient transfection with TLR-4 induced a marked increase in PU.1 mRNA expression in all of the conditions on RT-PCR (Figure 3d).

As PU.1 drives macrophage differentiation, the primary chorioamniotic cells were transiently transfected with fulllength PU.1 cDNA. Overexpression of PU.1 upregulated CD14 mRNA (Figure 4a) and protein expression (Figure 4b). CD14 promoter region analysis revealed four putative PU.1 binding motifs (consensus GAGGAA) within $600 \mathrm{bp}$ upstream of the ATG site (starting positions: $-200,-224$, -272 , and -379 ). Reporter gene analysis and ChIP assay were also performed. Among the four putative binding mo- tifs for PU.1 identified within the CD14 promoter region, a series of $5^{\prime}$-deletion mutant analysis localized a strong cis-acting element between nucleotides $-272 \mathrm{bp}$ and $-267 \mathrm{bp}$ upstream of the translation starting site (Figure 5a). A ChIP assay confirmed PU.1 binding in the CD14 promoter region (Figure 5b).

\section{DISCUSSION}

It is evident that the uterine cavity is not sterile, and microbial invasion of the amniotic cavity is not rare. ${ }^{25}$ The chorioamniotic membranes are, therefore, expected to have basic immunologic competency. However, the lack of fetal vessels and impervious nature of the chorioamniotic mesodermal layer intrinsically block the mobilization or trafficking of fetal leukocytes in the chorioamniotic membranes. ${ }^{7}$ The primary finding of this study is that 


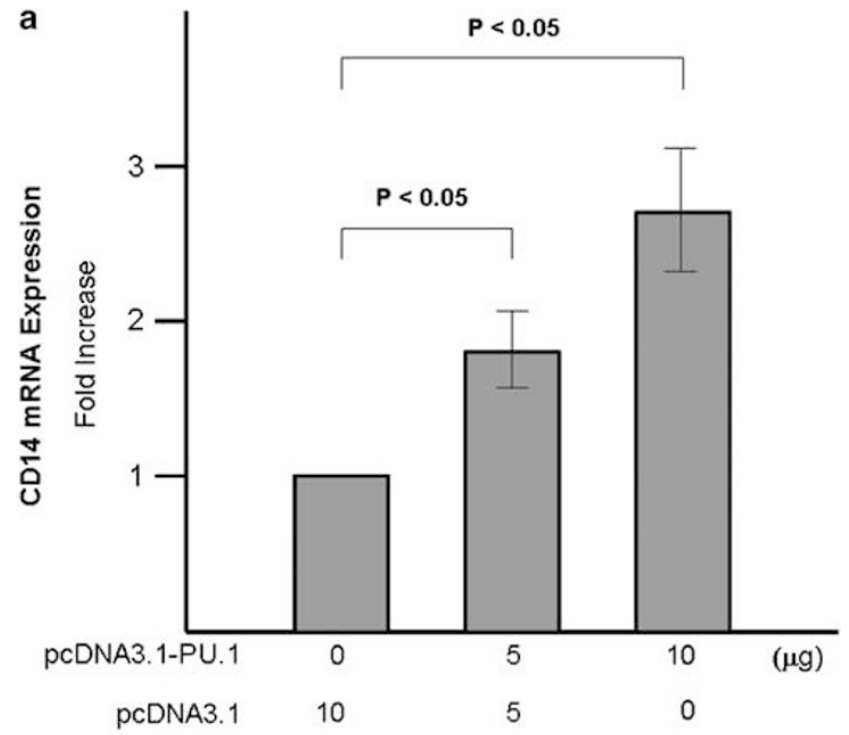

b

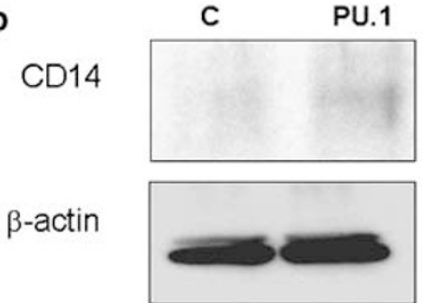

Figure 4 Chorionic mesodermal cells and the regulation of CD14 mRNA expression by PU.1. The cells were obtained by sequential treatment of chorion-decidua with dispase II and collagenase. (a) CD14 mRNA expression is increased in the chorionic mesodermal cells following transient transfection with full-length human PU.1 cDNA. CD14 mRNA expression was evaluated using mesodermal cells transfected with PU.1 expression vector at various concentrations for $24 \mathrm{~h}$ by qRT-PCR using exon-specific primers and normalization to $\beta$-actin mRNA. (b) Immunoblotting of PU.1-transfected cells showing increased CD14 expression following PU.1 expression.

chorionic mesodermal fibroblasts/myofibroblasts function as a reservoir of fetal tissue macrophages. This plasticity seems to be a quite relevant mechanism for ensuring diverse biological functions of the chorioamniotic membranes with a limited pool of cells.

A substantial body of evidence indicates that cellular compartments of the chorioamniotic membranes engage in immune response. Toll-like receptor- 2 and -4 are expressed in the chorioamniotic membranes, and their expression is increased in acute chorioamnionitis. ${ }^{26}$ In addition to the fact that acquisition of the macrophage phenotype by chorioamniotic mesodermal cells is an effective innate immune defense mechanism, it is also strongly suggested that expected biological changes with macrophage differentiation, such as decreased collagen synthesis and increased secretion of MMPs, could be linked to both the physiologic and pathologic rupture of the membranes. ${ }^{13,27}$ Concomitant expression of fibroblast/myofibroblast and macrophage markers in many cells coupled with sparse Ki-67 labeling makes it clear that a

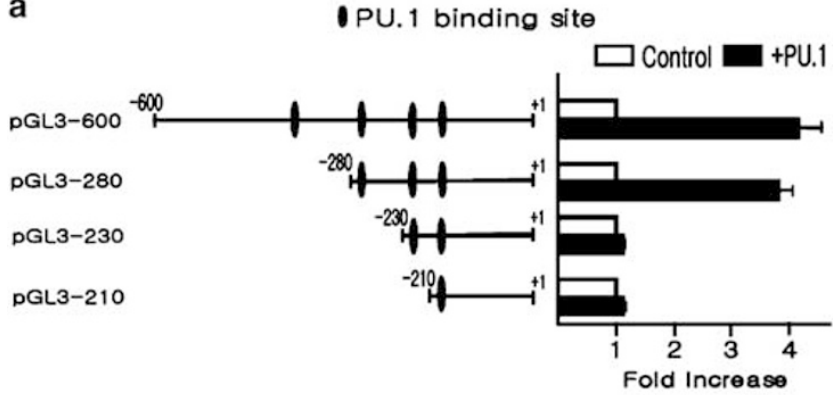

b

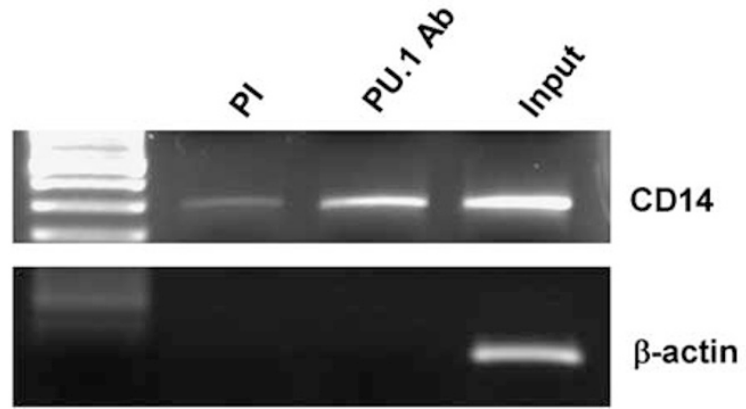

Figure $55^{\prime}$-Deletion analysis of promoter activity of CD14 gene and chromatin immunoprecipitation (ChIP) assay. The cells were obtained by sequential treatment of chorion-decidua with dispase II and collagenase. (a) A schematic diagram representing the proximal promoter region of CD14 gene from nucleotide -600 to +1 . The translation start site (ATG) is designated as +1 . Fragments of various lengths of the promoter region were inserted into the promoterless luciferase vector, pGL3-basic (Promega). The numbers on the left of each construct refer to the number of the nucleotide at the $5^{\prime}$ end of each construct, which is $-600 \mathrm{bp}$.

Transient transfections and luciferase assays of various constructs were performed. The promoter activity of each construct was expressed as $n$-fold activation of $\mathrm{pGL} 3$-basic promoter activity. The histograms represent the mean values of fold activation from three independent transfection experiments. Error bars indicate the range of mean \pm s.d. (b) ChIP assay was performed in mesodermal cells using anti-PU.1 antibody. Lysates from cells were immunoprecipitated with PU.1 antibody and nonspecific antibodies (preimmune serum) as a control, and the bound DNA was analyzed by PCR using primers that amplified a $280 \mathrm{bp}$ region of the CD14 promoter spanning the PU.1 binding site. PI: preimmune serum, Input: input control.

macrophages originate from de novo transdifferentiation of the mesodermal cells, not merely from the proliferation of existing resident macrophages. The changes in the proportion of fibroblasts/myofibroblasts and macrophages can reasonably explain the discrepancies surrounding the major subset of cells in the chorioamniotic mesodermal layer. ${ }^{14-16,18}$

Studies show that human chorioamniotic mesenchymal cells have the characteristics of mesenchymal stem cells (MSCs) differentiating into neurogenic, adipogenic, osteogenic, chondrogenic, and myogenic lineages. ${ }^{28,29}$ The International Society of Cellular Therapy (ISCT) recently proposed minimal criteria for the definition of MSCs, which include a lack of expression of CD45, CD34, CD14, or CD11b, CD79 $\alpha$ or CD19 and HLA-DR. ${ }^{30}$ Therefore, the immunophenotype of cells undergoing macrophage differentiation is not consistent with that of MSCs. Ets family transcription factor PU.1 is also selectively expressed 
a

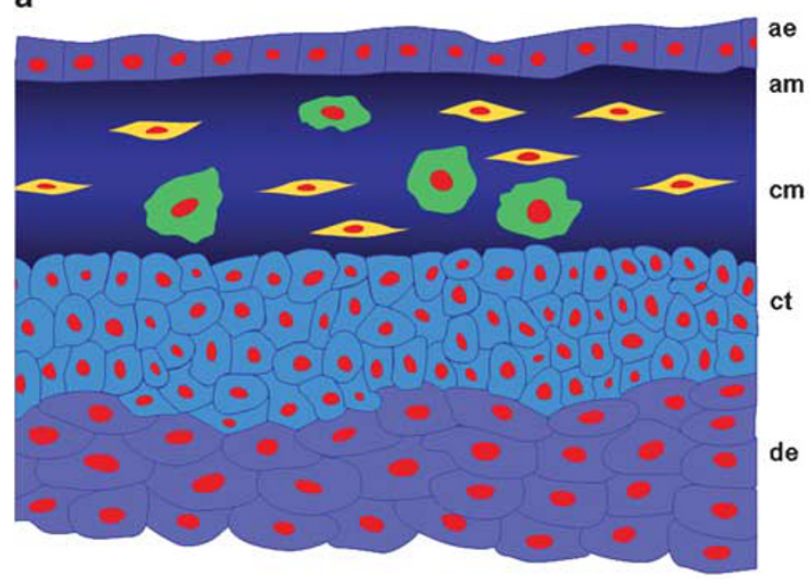

b

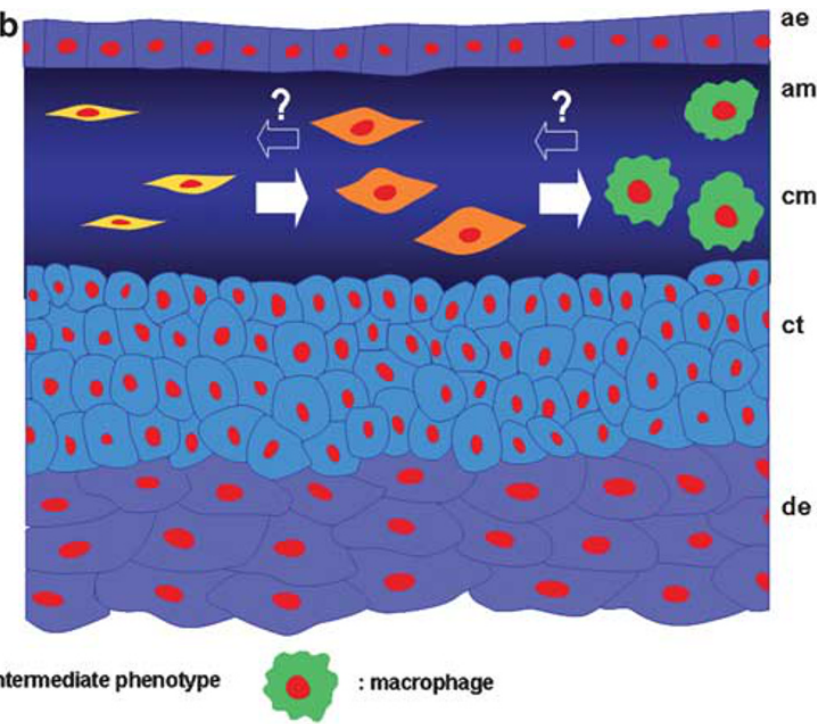

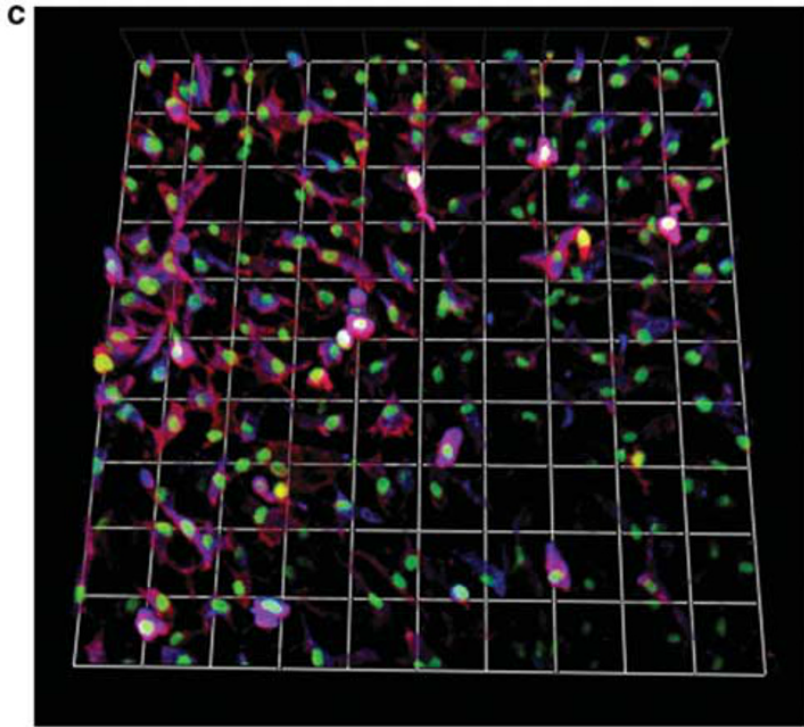

Figure 6 A new transdifferentiation model of chorioamiotic mesodermal cells. (a) A cartoon showing a conventional model. The chorioamniotic mesoderm contains two groups of cells. Fibroblasts/myofibroblasts are shown as spindle cells in the chorioamniotic mesoderm whereas macrophages are shown as round cells. (b) A cartoon showing a new model: a transdifferentiation sequence of fibroblast/myofibroblast to macrophage. Arrows here indicate putative differentiation pathways. Question marks indicate that the presence of differentiation pathway from macrophage to fibroblast/myofibroblast is uncertain. The balance between fibroblasts/myofibroblasts and macrophages may be affected by many physiologic and pathologic signals. ae: amnion epithelium, am: amnionic mesoderm, cm: chorionic mesoderm, ct: chorionic trophoblasts, de: decidua. (c) A Z-stack image of the amniotic mesodermal layer from a case of a term in labor patient showing variable degrees of isolated or concomitant expression of macrophage marker (CD163, mouse monoclonal antibody, redAlexa Fluor 555; Vector Laboratories) and smooth muscle marker ( $\alpha$ SMA, rabbit monoclonal antibody, blue-Alexa Fluor 633; Abcam). A piece of unsectioned amnion was fixed with graded ethanol and stained. The image was obtained using the Zeiss LSM 510 Meta laser scanning confocal microscope (Carl Zeiss Microimaging Inc., Germany).

in hematopoietic cells, such as mature macrophages, and increased PU.1 expression determines cell fate into macrophages. ${ }^{31}$ The expression of PU.1 in chorioamniotic mesodermal cells, as well as PU.1 binding in the promoter region of $\mathrm{CD} 14$, indicates that these cells are of hematopoietic lineage, and PU.1 plays a role in the macrophage differentiation. Frequent overlapping of procollagen, desmin, and CD163 immunoreactivity indicates that these cells have an intermediate phenotype between myofibroblasts and macrophages. PU.1 upregulates CD14, a major LPS receptor associated with TLR-4 and present in the cells of the monocyte-macrophage lineage. ${ }^{32}$ More interestingly, it was shown that mechanical deformation induces PU.1 in human monocytes/macrophages with selective augmentation of MMPs and induction of immediate early genes, suggesting a close link between PU.1 expression and proteolytic activity of macrophages, as well as a role in monocyte differentiation. ${ }^{33}$ 
Indeed, several studies have reported examples of immunophenotypic switching between myofibroblasts and monocytes/macrophages. Peripheral blood monocytes have been shown to acquire myofibroblastic characteristics during de novo granulation tissue formation; ${ }^{34,35}$ treatment of macrophages with TGF- $\beta 1$ induced transdifferentiation into smooth muscle-like cells expressing higher levels of $\alpha \mathrm{SMA}$ and calponin and lower levels of CD11 b; ${ }^{36}$ and aortic smooth muscle cells loaded with cholesterol were characterized by a decrease in $\alpha \mathrm{SMA}$ and $\alpha$-tropomyosin and increased levels of the macrophage markers CD68 and Mac-2 antigen. ${ }^{37}$ Circulating fibrocytes, which rapidly enter the sites of tissue injury and synthesize connective tissue matrix, are another population of blood-borne cells that have a distinct cell surface phenotype (collagen $1+/ \mathrm{CD} 13+/ \mathrm{CD} 34+/$ $\mathrm{CD} 45+$ ). They also express class II MHC molecules ${ }^{38}$ and are mesenchymal precursors of a monocyte/macrophage lineage. ${ }^{39}$ Monocyte-derived multipotential cells share an almost identical immunophenotype with fibrocytes. ${ }^{40}$

Although the results of this study do not rule out the possibility that chorioamniotic mesoderm is populated by both mesenchymal stem cells and cells of hematopoietic lineage, extensive and concomitant expression of fibroblast/ myofibroblast and macrophage markers in certain cases indicates that substantial proportions of the cells have plasticity. It is also of note that differentiation of CD14+ monocytes into diverse types of mesenchymal cells has been clearly documented. ${ }^{41,42}$ Based on our observations, we propose a new differentiation model of the chorioamiotic mesodermal cells (Figure $6 \mathrm{a}$ and $\mathrm{b}$ ), one that takes into account the coexpression of fibroblast/myofibroblast and macrophage markers and wherein proinflammatory responses are likely to shift the balance towards the macrophage spectrum. We think that the model explains previous discrepancies about the major cell population of the chorioamniotic mesodermal layer. Variable coexpression of CD163 and $\alpha \mathrm{SMA}$ in a Z-stack image of the amniotic mesodermal layer obtained from a term labor case also supports our hypothesis (Figure 6c).

In conclusion, we report that the chorioamniotic mesodermal cells are endowed with unique plasticity. They are the sole population of cells that change phenotype in the human chorioamniotic membranes. This feature would confer upon the cells the capacity to maintain or interrupt membrane integrity and deploy innate immunity. Further investigation of the biological signals controlling plasticity of the cells in the chorioamniotic mesoderm and the potential routing of the cells or histogenesis during development, will provide new insights into the role for these unique cells at the fetomaternal interface in association with physiologic and pathologic aspects of human pregnancy and parturition.

\section{ACKNOWLEDGEMENT}

This research was supported by the Intramural Research Program of the National Institute of Child Health and Human Development, NIH, DHHS.

\section{CONFLICT OF INTEREST}

The authors state no conflict of interest.

1. van Rozendaal BA, van Golde LM, Haagsman HP. Localization and functions of SP-A and SP-D at mucosal surfaces. Pediatr Pathol Mol Med 2001;20:319-339.

2. van Spreeuwel JP, Lindeman J, Meijer CJ. Quantitative analysis of immunoglobulin-containing cells in gastrointestinal pathology. Anal Quant Cytol Histol 1986;8:314-320.

3. Eckmann L, Kagnoff MF. Intestinal mucosal responses to microbial infection. Springer Semin Immunopathol 2005;27:181-196.

4. Kim YM, Romero $R$, Chaiworapongsa $T$, et al. Dermatitis as a component of the fetal inflammatory response syndrome is associated with activation of Toll-like receptors in epidermal keratinocytes. Histopathology 2006;49:506-514.

5. Kumar NB, Nostrant TT, Appelman HD. The histopathologic spectrum of acute self-limited colitis (acute infectious-type colitis). Am J Surg Pathol 1982;6:523-529.

6. Millar LK, Stollberg J, DeBuque L, et al. Fetal membrane distention: determination of the intrauterine surface area and distention of the fetal membranes preterm and at term. Am J Obstet Gynecol 2000;182:128-134.

7. Hoyes $A D$. Ultrastructure of the mesenchymal layers of the human chorion laeve. J Anat 1971;109:17-30.

8. Romero R, Sirtori M, Oyarzun E, et al. Infection and labor. V. Prevalence, microbiology, and clinical significance of intraamniotic infection in women with preterm labor and intact membranes. Am J Obstet Gynecol 1989;161:817-824.

9. Yoon $\mathrm{BH}$, Romero $\mathrm{R}$, Moon JB, et al. Clinical significance of intraamniotic inflammation in patients with preterm labor and intact membranes. Am J Obstet Gynecol 2001;185:1130-1136.

10. Redline RW. Inflammatory responses in the placenta and umbilical cord. Semin Fetal Neonatal Med 2006;11:296-301.

11. Redline RW, Faye-Petersen $\mathrm{O}$, Heller $\mathrm{D}$, et al. Amniotic infection syndrome: nosology and reproducibility of placental reaction patterns. Pediatr Dev Pathol 2003;6:435-448.

12. Bryant-Greenwood GD. The extracellular matrix of the human fetal membranes: structure and function. Placenta 1998;19:1-11.

13. Parry S, Strauss III JF. Premature rupture of the fetal membranes. N Engl J Med 1998;338:663-670.

14. McParland PC, Taylor DJ, Bell SC. Myofibroblast differentiation in the connective tissues of the amnion and chorion of term human fetal membranes-implications for fetal membrane rupture and labour. Placenta 2000;21:44-53.

15. Sutton L, Mason DY, Redman CW. HLA-DR positive cells in the human placenta. Immunology 1983;49:103-112.

16. Bulmer JN, Johnson PM. Macrophage populations in the human placenta and amniochorion. Clin Exp Immunol 1984;57:393-403.

17. Jenkins DM, O'Neill M, Johnson PM. HLA-DR-positive cells in the human amniochorion. Immunol Lett 1983;6:65-67.

18. Wang T, Schneider J. Fine structure of human chorionic membrane. Ultrastructural and histochemical examinations. Arch Gynecol 1983;233:187-198.

19. Malak TM, Bell SC. Structural characteristics of term human fetal membranes: a novel zone of extreme morphological alteration within the rupture site. Br J Obstet Gynaecol 1994;101:375-386.

20. Kruidenier L, MacDonald TT, Collins JE, et al. Myofibroblast matrix metalloproteinases activate the neutrophil chemoattractant CXCL7 from intestinal epithelial cells. Gastroenterology 2006;130:127-136.

21. Ronty MJ, Leivonen SK, Hinz B, et al. Isoform-specific regulation of the actin-organizing protein palladin during TGF-beta1-Induced myofibroblast differentiation. J Invest Dermatol 2006;126:2387-2396.

22. Smith PD, Ochsenbauer-Jambor C, Smythies LE. Intestinal macrophages: unique effector cells of the innate immune system. Immunol Rev 2005;206:149-159.

23. Lloberas J, Soler C, Celada A. The key role of PU.1/SPI-1 in B cells, myeloid cells and macrophages. Immunol Today 1999;20:184-189.

24. Dakic A, Metcalf D, Di Rago L, et al. PU.1 regulates the commitment of adult hematopoietic progenitors and restricts granulopoiesis. J Exp Med 2005;201:1487-1502.

25. Romero R, Espinoza J, Mazor M. Can endometrial infection/ inflammation explain implantation failure, spontaneous abortion, 
and preterm birth after in vitro fertilization? Fertil Steril 2004;82:799804.

26. Kim YM, Romero R, Chaiworapongsa T, et al. Toll-like receptor-2 and -4 in the chorioamniotic membranes in spontaneous labor at term and in preterm parturition that are associated with chorioamnionitis. Am J Obstet Gynecol 2004;191:1346-1355.

27. Tetley TD. Macrophages and the pathogenesis of COPD. Chest 2002;121:156S-159S.

28. Portmann-Lanz CB, Schoeberlein A, Huber A, et al. Placental mesenchymal stem cells as potential autologous graft for pre- and perinatal neuroregeneration. Am J Obstet Gynecol 2006;194:664-673.

29. Sakuragawa N, Kakinuma K, Kikuchi $A$, et al. Human amnion mesenchyme cells express phenotypes of neuroglial progenitor cells. J Neurosci Res 2004;78:208-214.

30. Dominici M, Le Blanc K, Mueller I, et al. Minimal criteria for defining multipotent mesenchymal stromal cells. The international society for cellular therapy position statement. Cytotherapy 2006; 8:315-317.

31. Laslo $\mathrm{P}$, Spooner $\mathrm{CJ}$, Warmflash $\mathrm{A}$, et al. Multilineage transcriptional priming and determination of alternate hematopoietic cell fates. Cell 2006;126:755-766.

32. Berclaz PY, Carey B, Fillipi MD, et al. GM-CSF regulates a PU.1dependent transcriptional program determining the pulmonary response to LPS. Am J Respir Cell Mol Biol 2007;36: 114-121.

33. Yang JH, Sakamoto $\mathrm{H}, \mathrm{Xu} E \mathrm{EC}$, et al. Biomechanical regulation of human monocyte/macrophage molecular function. Am J Pathol 2000;156:1797-1804.
34. Jabs A, Moncada GA, Nichols CE, et al. Peripheral blood mononuclear cells acquire myofibroblast characteristics in granulation tissue. J Vasc Res 2005;42:174-180.

35. Abe R, Donnelly SC, Peng $T$, et al. Peripheral blood fibrocytes: differentiation pathway and migration to wound sites. J Immunol 2001;166:7556-7562.

36. Ninomiya $\mathrm{K}$, Takahashi A, Fujioka $Y$, et al. Transforming growth factorbeta signaling enhances transdifferentiation of macrophages into smooth muscle-like cells. Hypertens Res 2006;29:269-276.

37. Rong JX, Shapiro M, Trogan E, et al. Transdifferentiation of mouse aortic smooth muscle cells to a macrophage-like state after cholesterol loading. Proc Natl Acad Sci USA 2003;100:13531-13536.

38. Chesney J, Bacher M, Bender A, et al. The peripheral blood fibrocyte is a potent antigen-presenting cell capable of priming naive $T$ cells in situ. Proc Natl Acad Sci USA 1997;94:6307-6312.

39. Frid MG, Brunetti JA, Burke DL, et al. Hypoxia-induced pulmonary vascular remodeling requires recruitment of circulating mesenchymal precursors of a monocyte/macrophage lineage. Am J Pathol 2006;168:659-669.

40. Kuwana $\mathrm{M}$, Okazaki $\mathrm{Y}$, Kodama $\mathrm{H}$, et al. Endothelial differentiation potential of human monocyte-derived multipotential cells. Stem Cells 2006;24:2733-2743.

41. Kuwana $M$, Okazaki $\mathrm{Y}$, Kodama $\mathrm{H}$, et al. Human circulating CD14+ monocytes as a source of progenitors that exhibit mesenchymal cell differentiation. J Leukoc Biol 2003;74:833-845.

42. Kodama $H$, Inoue $T$, Watanabe $R$, et al. Neurogenic potential of progenitors derived from human circulating CD14+ monocytes. Immunol Cell Biol 2006;84:209-217. 